\title{
A MODULE INDUCED FROM A WHITTAKER MODULE
}

\author{
EDWARD MCDOWELL
}

(Communicated by Maurice Auslander)

\begin{abstract}
In an earlier paper [On modules induced from Whittaker modules, J. Algebra 96 (1985)] we constructed a class of induced modules, over a finitedimensional semisimple Lie algebra, which includes the Verma modules of Verma [Structure of certain induced representations of complex semisimple Lie algebras, Bull. Amer. Math. Soc. 74 (1968)] and the irreducible Whittaker modules of Kostant [On Whittaker vectors and representation theory, Invent. Math. 48 (1978)]. We proved that every module in this class has finite length and is irreducible most of the time. In this article we present a concrete example of this construction, over $\operatorname{sl}(3, C)$, showing that proper submodules can exist when the induced module is not a Verma module.
\end{abstract}

Let $\mathfrak{g}$ be a finite-dimensional semisimple Lie algebra over $\mathbb{C}$. Let $\mathfrak{h}$ be a Cartan subalgebra and let $\mathfrak{n}$ be the sum of the positive root spaces corresponding to a suitable fundamental set of roots. A family of $\mathfrak{g}$-modules $M_{\Omega, c}$ was constructed in [2], where $c$ is a Lie algebra homomorphism taking $\mathfrak{n}$ into $\mathbb{C}$ and $\Omega$ is a central character for a certain reductive subalgebra of $\mathfrak{g}$ determined by $c$. This family includes both the Verma modules of [3] and the irreducible Whittaker modules of [1]. It was shown in [2] that each $M_{\Omega, c}$ has a unique irreducible quotient and that every irreducible $\mathfrak{n}$-finite $\mathfrak{g}$-module is isomorphic to one of these quotients. It was shown in Theorem 2.15 of [2] that $M_{\Omega, c}$ is itself irreducible for most choices of $c$ and $\Omega$. The proof of this assertion was abstract and did not show conclusively that $M_{\Omega, c}$ could have proper submodules in the important case where $M_{\Omega, c}$ is neither a Verma module nor an irreducible Whittaker module. We present a concrete example of this construction here for $\mathfrak{g}=\operatorname{sl}(3, \mathbb{C})$. We derive an explicit equation involving $\Omega$ and $c$ that can be used to determine whether $M_{\Omega, c}$ has proper submodules. We show that proper submodules can exist and display explicit vectors that generate them.

We fix $\mathfrak{g}=\operatorname{sl}(3, \mathbb{C})$, the Lie algebra of $3 \times 3$ matrices over $\mathbb{C}$ having trace zero, and let $x_{1}=e_{12}, y_{1}=e_{21}, h_{1}=e_{11}-e_{22}, x_{2}=e_{23}, y_{2}=e_{32}, h_{2}=$ $e_{22}-e_{33}, x_{3}=e_{13}, y_{3}=e_{31}, h_{3}=e_{11}-e_{33}=h_{1}+h_{2}$, and $z=e_{11}+e_{22}-2 e_{33}=$ $h_{1}+2 h_{2}$. Then $y_{3}, y_{2}, y_{1}, h_{1}, h_{2}, x_{1}, x_{2}$, and $x_{3}$ form a basis for $\mathfrak{g}$ over $\mathbb{C}$ and the Lie commutator operation is as shown in Table 1. Using this table and induction on $k$, we may verify the following lemma.

Received by the editors September 27, 1991.

1991 Mathematics Subject Classification. Primary 17B10. 
TABLE 1. Lie commutator for $\operatorname{sl}(3, \mathbb{C})$

\begin{tabular}{|c|c|c|c|c|c|c|c|c|c|}
\hline & & & & $w$ & & & & & \\
\hline & & $y_{3}$ & $y_{2}$ & $y_{1}$ & $h_{1}$ & $h_{2}$ & $x_{1}$ & $x_{2}$ & $x_{3}$ \\
\hline & $y_{3}$ & 0 & 0 & 0 & $y_{3}$ & $y_{3}$ & $y_{2}$ & $-y_{1}$ & $x_{3}$ \\
\hline & $y_{2}$ & 0 & 0 & $y_{3}$ & $-y_{2}$ & $2 y_{2}$ & 0 & $-h_{2}$ & $-x_{1}$ \\
\hline & $y_{1}$ & 0 & $-y_{3}$ & 0 & $2 y_{1}$ & $-y_{1}$ & $-h_{1}$ & 0 & $x_{2}$ \\
\hline$v$ & $h_{1}$ & $-y_{3}$ & $y_{2}$ & $-2 y_{1}$ & 0 & 0 & $2 x_{1}$ & $-x_{2}$ & $x_{3}$ \\
\hline & $h_{2}$ & $-y_{3}$ & $-2 y_{2}$ & $y_{1}$ & 0 & 0 & $-x_{1}$ & $2 x_{2}$ & $x_{3}$ \\
\hline & $x_{1}$ & $-y_{2}$ & 0 & $h_{1}$ & $-2 x_{1}$ & $x_{1}$ & 0 & $x_{3}$ & 0 \\
\hline & $x_{2}$ & $y_{1}$ & $h_{2}$ & 0 & $x_{2}$ & $-2 x_{2}$ & $-x_{3}$ & 0 & 0 \\
\hline & $x_{3}$ & $h_{3}$ & $x_{1}$ & $-x_{2}$ & $-x_{3}$ & $-x_{3}$ & 0 & 0 & 0 \\
\hline
\end{tabular}

Lemma 1. The following equations hold in the universal enveloping algebra $U(\mathfrak{g})$ of $\mathfrak{g}$ for every positive integer $k$.

$$
\begin{gathered}
x_{1} y_{2}^{k}=y_{2}^{k} x_{1} . \\
x_{1} h_{1}^{k}=\left(h_{1}-2\right)^{k} x_{1} . \\
y_{1} h_{1}^{k}=\left(h_{1}+2\right)^{k} y_{1} . \\
x_{2} y_{3}^{k}=y_{3}^{k} x_{2}+k y_{3}^{k-1} y_{1} . \\
x_{3} y_{2}^{k}=y_{2}^{k} x_{3}+k y_{2}^{k-1} x_{1} . \\
x_{1} y_{3}^{k}=y_{3}^{k} x_{1}-k y_{3}^{k-1} y_{2} . \\
y_{1} y_{2}^{k}=y_{2}^{k} y_{1}-k y_{3} y_{2}^{k-1} . \\
h_{3} y_{2}^{k}=y_{2}^{k} h_{3}-k y_{2}^{k} . \\
x_{2} y_{2}^{k}=y_{2}^{k} x_{2}+k y_{2}^{k-1} h_{2}-k(k-1) y_{2}^{k-1} . \\
x_{3} y_{3}^{k}=y_{3}^{k} x_{3}+k y_{3}^{k-1} h_{3}-k(k-1) y_{3}^{k-1} .
\end{gathered}
$$

$\mathfrak{g}$ has the Cartan subalgebra $\mathfrak{h}=\mathbb{C} h_{1}+\mathbb{C} h_{2}$. Define $\rho_{i j} \in \mathfrak{h}^{*}$ by $\rho_{i j}\left(a_{1} e_{11}+a_{2} e_{22}+a_{3} e_{33}\right)=a_{i}-a_{j}$. Then $\rho_{12}, \rho_{23}, \rho_{13}, \rho_{21}, \rho_{32}, \rho_{31}$ are the roots of $\mathfrak{g}$ with respect to $\mathfrak{h}$ having root vectors $x_{1}, x_{2}, x_{3}, y_{1}, y_{2}, y_{3}$, respectively. $\Delta=\left\{\rho_{12}, \rho_{23}\right\}$ is a fundamental set of roots and $\mathfrak{n}=\mathbb{C} x_{1}+\mathbb{C} x_{2}+\mathbb{C} x_{3}$ is the corresponding sum of the positive root spaces.

Now we fix a nonzero complex number $\alpha$ and consider the Lie algebra homomorphism $c: \mathfrak{n} \rightarrow \mathbb{C}$ for which $c\left(x_{1}\right)=\alpha, c\left(x_{2}\right)=0$, and $c\left(x_{3}\right)=0$. This homomorphism is singular, in the sense of [1], since it annihilates the fundamental root vector $x_{2}$, but it is not identically zero. As in Proposition 1.7 of [2], $\mathfrak{l}$ is the reductive subalgebra of $\mathfrak{g}$ generated by $\mathfrak{h}$, the fundamental root spaces on which $c$ does not vanish, and the corresponding negative root spaces. In our example $\mathfrak{l}=\mathbb{C} y_{1}+\mathbb{C} h_{1}+\mathbb{C} h_{2}+\mathbb{C} x_{1}$. Letting $z=h_{1}+2 h_{2}$, we have the decomposition $\mathfrak{l}=\mathfrak{s} \oplus \mathfrak{z}$ where $\mathfrak{s}=\mathbb{C} y_{1}+\mathbb{C} h_{1}+\mathbb{C} x_{1}$ is isomorphic to $\operatorname{sl}(2, \mathbb{C})$ and $\mathfrak{z}=\mathbb{C} z$ is the center of $\mathfrak{l}$. We also have $\mathfrak{g}=\overline{\mathfrak{m}}+\mathfrak{l}+\mathfrak{m}$ where $\mathfrak{m}=\mathbb{C} x_{2}+\mathbb{C} x_{3}$ 
and $\overline{\mathfrak{m}}=\mathbb{C} y_{3}+\mathbb{C} y_{2}$ are nilpotent (in this case abelian) subalgebras of $\mathfrak{g}$. We let $\mathfrak{p}$ denote the parabolic subalgebra $\mathfrak{l}+\mathfrak{m}$.

We let $Z(\mathfrak{s})$ and $Z(\mathfrak{l})$ denote the centers of the enveloping algebras $U(\mathfrak{s})$ and $U(\mathfrak{l})$, respectively. Then $Z(\mathfrak{l})$ is isomorphic to $Z(\mathfrak{s}) \otimes U(\mathfrak{z})$ by the PBW Theorem. $Z(\mathfrak{s})$ and $U(\mathfrak{z})$ are each polynomial algebras generated by the indeterminates $w=4 y_{1} x_{1}+h_{1}^{2}+2 h_{1}$ and $z$, respectively. It follows that $Z(\mathfrak{l})$ is a polynomial algebra generated by the two indeterminates $w$ and $z$.

Given a central character $\Omega: Z(\mathfrak{l}) \rightarrow \mathbb{C}$, we now construct the induced module $M_{\Omega, c}$ as in Proposition 2.4 of [2]. Evidently $\Omega$ is determined by the parameters $\beta=\Omega(z)$ and $\gamma=\Omega(w)$. We begin by constructing the irreducible Whittaker module $Y_{\bar{\Omega}, \bar{c}}$ over $\mathfrak{s}$, where $\bar{\Omega}$ is the restriction of $\Omega$ to $Z(\mathfrak{s})$ and $\bar{c}$ is the restriction of $c$ to $\mathbb{C} x_{1}$. This module, constructed formally in Theorem 3.6.1 of [1], is the unique $\mathfrak{s}$-module having central character $\bar{\Omega}$ and generated by a nonzero vector $v_{0}$ for which $x_{1} v_{0}=\alpha v_{0}$. The following lemma provides a useful basis for this module.

Lemma 2. The $h_{1}^{k} v_{0}$, for $k \in \mathbb{N}$, form a basis for $Y_{\bar{\Omega}, \bar{c}}$ over $\mathbb{C}$.

Proof. To show that this set spans $Y_{\bar{\Omega}, \bar{c}}$ it suffices to show that it is stable under the action of $\mathfrak{s}$. Since

$$
\gamma v_{0}=w v_{0}=\left(4 y_{1} x_{1}+h_{1}^{2}+2 h_{1}\right) v_{0}=4 \alpha y_{1} v_{0}+h_{1}^{2} v_{0}+2 h_{1} v_{0},
$$

it follows that

$$
y_{1} v_{0}=\frac{1}{4 \alpha}\left(\gamma-2 h_{1}-h_{1}^{2}\right) v_{0} .
$$

It follows easily from (2) and (3) of Lemma 1 that

$$
x_{1} h_{1}^{k} v_{0}=\left(h_{1}-2\right)^{k} x_{1} v_{0}=\alpha\left(h_{1}-2\right)^{k} v_{0}
$$

and

$$
y_{1} h_{1}^{k} v_{0}=\left(h_{1}+2\right)^{k} y_{1} v_{0}=\frac{1}{4 \alpha}\left(h_{1}+2\right)^{k}\left(\gamma-2 h_{1}-h_{1}^{2}\right) v_{0}
$$

for each nonnegative integer $k$ so the span of the $h_{1}^{k} v_{0}$ is $\mathbf{s}$-stable. Finally, the $h_{1}^{k} v_{0}$ are linearly independent; otherwise, they would span a finite-dimensional s-module and we would have $x_{1}^{n} v_{0}=0$ for suitably large $n$. This is impossible since $x_{1}^{n} v_{0}=\alpha^{n} v_{0} \neq 0$.

We extend the action of $\mathfrak{s}$ on $Y_{\bar{\Omega}, \bar{c}}$ to an action of $\mathfrak{p}$ by requiring that $z v=\beta v, x_{2} v=0, x_{3} v=0$ for each $v \in Y_{\bar{\Omega}, \bar{c}} . M_{\Omega, c}$ is then the induced $\mathfrak{g}$-module $U(\mathfrak{g}) \otimes_{U(\mathfrak{p})} Y_{\bar{\Omega}, \bar{c}}$. The following lemma, which parallels Proposition 2.4 of [2], lists some basic properties of $M_{\Omega, c}$.

Lemma 3. Properties of $M_{\Omega, c}$.

(a) $M_{\Omega, c}$ is generated as a $\mathfrak{g}$-module by $1 \otimes v_{0}$. From here on $v_{0}$ denotes this vector. C.

(b) The vectors $y_{3}^{k_{3}} y_{2}^{k_{2}} h_{1}^{k_{1}} v_{0}$, for $k_{1}, k_{2}, k_{3} \in \mathbb{N}$, form a basis for $M_{\Omega, c}$ over

(c) Given $\lambda \in \mathbb{C}$, let $M_{\Omega, c}^{\lambda}=\left\{v \in M_{\Omega, c} \mid z v=\lambda v\right\}$. Then each $M_{\Omega, c}^{\lambda}$ is Istable, $y_{2} M_{\Omega, c}^{\lambda} \subset M_{\Omega, c}^{\lambda-3}, y_{3} M_{\Omega, c}^{\lambda} \subset M_{\Omega, c}^{\lambda-3}, x_{2} M_{\Omega, c}^{\lambda} \subset M_{\Omega, c}^{\lambda+3}, x_{3} M_{\Omega, c}^{\lambda} \subset M_{\Omega, c}^{\lambda+3}$, and $y_{3}^{k_{3}} y_{2}^{k_{2}} h_{1}^{k_{1}} v_{0} \in M_{\Omega, c}^{\beta-3 k_{2}-3 k_{3}}$. 
(d) $\mathfrak{z}$ acts semisimply on $M_{\Omega, c}, M_{\Omega, c}=\bigoplus_{k=0}^{\infty} M_{\Omega, c}^{\beta-3 k}$, and $M_{\Omega, c}^{\beta}=U(\mathfrak{l}) v_{0}$ is irreducible as an l-module.

Proof. (a) follows directly from the construction. (b) follows from Lemma 2 since the $y_{3}^{k_{3}} y_{2}^{k_{2}}$ from a basis for $U(\mathfrak{g})$ as a free right $U(\mathfrak{p})$-module by the PBW theorem. (c) is easily verified. (d) follows from (c) and from the fact that $Y_{\bar{\Omega}, \bar{c}}$ is an irreducible s-module.

We now wish to determine when $M_{\Omega, c}$ contains proper submodules. As in [2], we shall call a nonzero vector $v$ in $M_{\Omega, c}$ for which $\mathfrak{m} v=0$ a maximal vector. The following lemma asserts that it suffices to seek maximal vectors.

Lemma 4. $M_{\Omega, c}$ contains a proper submodule iff there is a positive integer $n$ such that $M_{\Omega, c}^{\beta-3 n}$ contains a maximal vector.

Proof. Suppose that $M_{\Omega, c}^{\beta-3 n}$ contains a maximal vector $v$. Since $U(\mathfrak{g})=$ $U(\overline{\mathfrak{m}}) U(\mathfrak{l}) U(\mathfrak{m})$, by the PBW theorem, we have $U(\mathfrak{g}) v=U(\overline{\mathfrak{m}}) U(\mathfrak{l}) v$. It now follows from Lemma $3(\mathrm{c})$ that $U(\mathfrak{g}) v \subset \sum_{k \geq n} M_{\Omega, c}^{\beta-3 k}$, so $U(\mathfrak{g}) v$ is a proper submodule of $M_{\Omega, c}$. Now suppose that $N$ is a proper submodule of $M_{\Omega, c}$. Since $\mathfrak{z}$ acts semisimply on $M_{\Omega, c}$, we have $N=\sum_{k=0}^{\infty} N \cap M_{\Omega, c}^{\beta-3 k}$. Let $n$ be the smallest $k$ for which $N \cap M_{\Omega, c}^{\beta-3 k}$ is nontrivial. Since any nonzero vector in $M_{\Omega, c}^{\beta}$ generates $M_{\Omega, c}, n$ must be positive. It now follows from Lemma 3(c) and the minimality of $n$ that any nonzero vector in $N \cap M_{\Omega, c}^{\beta-3 n}$ is maximal.

It follows from Lemma 3 that there is a bijection between vectors $\hat{v}=$ $\sum_{i=0}^{n} y_{3}^{n-i} y_{2}^{i} P_{i}\left(h_{i}\right) v_{0}$ in $M_{\Omega, c}^{\beta-3 n}$ and sequences of polynomials $P_{0}(T), P_{1}(T)$, $\ldots, P_{n}(T)$ from $\mathbb{C}[T]$. The following lemma gives the action of $x_{1}, x_{2}$, and $x_{3}$ on the typical vector $\hat{v}$ of $M_{\Omega, c}^{\beta-3 n}$.

Lemma 5. Let $n$ be a positive integer and let $\hat{v}=\sum_{i=0}^{n} y_{3}^{n-i} y_{2}^{i} P_{i}\left(h_{1}\right) v_{0}$ be a typical vector in $M_{\Omega, c}^{\beta-3 n}$. Then

(a)

$$
x_{1} \hat{v}=\alpha y_{3}^{n} P_{0}\left(h_{1}-2\right) v_{0}+\sum_{i=1}^{n} y_{3}^{n-i} y_{2}^{i}\left(\alpha P_{i}\left(h_{1}-2\right)-(n-i+1) P_{i-1}\left(h_{1}\right)\right) v_{0} ;
$$

(b)

$$
\begin{aligned}
x_{2} \hat{v}=\sum_{i=1}^{n} y_{3}^{n-i} y_{2}^{i-1}\left[\frac{1}{4 \alpha}(n-i\right. & +1) P_{i-1}\left(h_{1}+2\right)\left(\gamma-2 h_{1}-h_{1}^{2}\right) \\
& \left.+i P_{i}\left(h_{1}\right)\left(\frac{1}{2} \beta-\frac{1}{2} h_{1}-n+1\right)\right] v_{0}
\end{aligned}
$$

(c)

$$
\begin{aligned}
x_{3} \hat{v}=\sum_{i=1}^{n} y_{3}^{n-i} y_{2}^{i-1}\left[i \alpha P_{i}\left(h_{1}-2\right)\right. & +(n-i+1) P_{i-1}\left(h_{1}\right) \\
\times & \left.\left(\frac{1}{2} \beta+\frac{1}{2} h_{1}-n+1\right)\right] v_{0} .
\end{aligned}
$$


Proof. (a) follows from direct computation using (6) and (1) of Lemma 1 and (11) from the proof of Lemma 2. (b) follows from (4), (9), and (7) of Lemma 1 , (12) from the proof of Lemma $2, \mathfrak{m} U(l) v_{0}=0$, and $h_{2} v_{0}=\left(\frac{1}{2} z-\frac{1}{2} h_{1}\right) v_{0}=$ $\left(\frac{1}{2} \beta-\frac{1}{2} h_{1}\right) v_{0}$. (c) follows from (10), (5), and (8) of Lemma 1, (11) from the proof of Lemma $2, \mathfrak{m} U(\mathfrak{l}) v_{0}=0$, and $h_{3} v_{0}=\left(\frac{1}{2} z+\frac{1}{2} h_{1}\right) v_{0}=\left(\frac{1}{2} \beta+\frac{1}{2} h_{1}\right) v_{0}$.

The following proposition states when $M_{\Omega, c}^{\beta-3 n}$ contains a maximal vector.

Proposition 1. Let $n$ be a positive integer. Then $M_{\Omega, c}^{\beta-3 n}$ contains a maximal vector iff $\beta=\Omega(z)$ and $\gamma=\Omega(w)$ satisfy the equation

$$
(2 n-\beta-3)^{2}=1+\gamma .
$$

Proof. Let $\hat{v}=\sum_{i=0}^{n} y_{3}^{n-i} y_{2}^{i} P_{i}\left(h_{1}\right)$ be a typical vector in $M_{\Omega, c}^{\beta-3 n}$. It follows from Lemma $5(\mathrm{c})$ that $x_{3} \hat{v}=0$ iff the equations $i \alpha P_{i}(T-2)+$ $(n-i+1) P_{i-1}(T)\left(\frac{1}{2} \beta+\frac{1}{2} T-n+1\right)=0$ hold in $\mathbb{C}[T]$ for $i=1,2, \ldots, n$. Applying the algebra automorphism of $\mathbb{C}[T]$ taking $T$ to $T+2$, we see that this is the case iff

$$
i \alpha P_{i}(T)+(n-i+1) P_{i-1}(T+2)\left(\frac{1}{2} \beta+\frac{1}{2} T-n+2\right)=0
$$

in $\mathbb{C}[T]$ for $i=1,2, \ldots, n$. Nonzero $\hat{v}$ in $M_{\Omega, c}^{\beta-3 n}$ for which $x_{3} \hat{v}=0$ can always be found by choosing an arbitrary nonzero polynomial $P_{0}(T)$ and then generating $P_{1}(T), P_{2}(T), \ldots, P_{n}(T)$ from (14). From Lemma 5(b) we have $x_{2} \hat{v}=0$ iff

$$
\begin{array}{r}
\frac{1}{4 \alpha}(n-i+1) P_{i-1}(T+2)\left(\gamma-2 T-T^{2}\right) \\
+i P_{i}(T)\left(\frac{1}{2} \beta-\frac{1}{2} T-n+1\right)=0
\end{array}
$$

in $\mathbb{C}[T]$ for $i=1,2, \ldots, n$. Thus $\hat{v}$ is maximal iff $P_{0}(T) \neq 0$ and equations (14) and (15) hold. Using equations (14) to substitute for $P_{i}(T)$ in terms of $P_{i-1}(T+2)$ in equations $(15)$, we have that $\hat{v}$ is maximal iff $P_{0}(T) \neq 0$, equations (14) hold, and

$$
\begin{aligned}
& \frac{1}{4 \alpha}(n-i+1) P_{i-1}(T+2)\left(\gamma-2 T-T^{2}\right) \\
& \quad+\frac{1}{\alpha}(n-i+1) P_{i-1}(T+2)\left(n-\frac{1}{2} \beta-\frac{1}{2} T-2\right)\left(\frac{1}{2} \beta-\frac{1}{2} T-n+1\right) \\
& \quad=0
\end{aligned}
$$

in $\mathbb{C}[T]$ for $i=1,2, \ldots, n$. It follows from this that $\hat{v}$ is maximal iff $P_{0}(T) \neq 0$, equations (14) hold, and

$$
\begin{aligned}
0 & =\gamma-2 T-T^{2}+4\left(n-\frac{1}{2} \beta-\frac{1}{2} T-2\right)\left(\frac{1}{2} \beta-\frac{1}{2} T-n+1\right) \\
& =\gamma-4 n^{2}+4 \beta n+12 n-\beta^{2}-6 \beta-8 \\
& =1+\gamma-4 n^{2}+4(\beta+3) n-(\beta+3)^{2} \\
& =1+\gamma-(2 n-\beta-3)^{2} .
\end{aligned}
$$

As in [2], a $c$-vector is a vector $v \in M_{\Omega, c}$ such that $x v=c(x) v$ for all $x \in \mathfrak{n}$. Clearly, every nonzero $c$-vector is maximal and a maximal vector $v$ is 
a $c$-vector iff $x_{1} v=\alpha v$. The following proposition determines the $c$-vectors of $M_{\Omega, c}^{\beta-3 n}$.

Proposition 2. Let $n$ be a positive integer. Then $M_{\Omega, c}^{\beta-3 n}$ contains a nonzero $c$-vector iff it contains a maximal vector. In this case the space of c-vectors is the one-dimensional vector space spanned by $\hat{v}=\sum_{i=0}^{n} y_{3}^{n-i} y_{2}^{i} P_{i}\left(h_{1}\right) v_{0}$ where $P_{0}(T)=1, \Lambda(T)=2 n-\beta-T-4$, and

$$
P_{i}(T)=\frac{n(n-1) \cdots(n-i+1)}{(2 \alpha)(4 \alpha) \cdots(2 i \alpha)} \Lambda(T) \Lambda(T+2) \cdots \Lambda(T+2 i-2)
$$

for $i=1,2, \ldots, n$.

Proof. Since every nonzero $c$-vector is maximal, one direction is obvious. Assume that $M_{\Omega, c}^{\beta-3 n}$ has a maximal vector. Then (13) holds and any vector $v=\sum_{i=0}^{n} y_{3}^{n-i} y_{2}^{i} P_{i}\left(h_{1}\right) v_{0}$ for which $P_{0}(T) \neq 0$ and equations (14) are satisfied is a maximal vector. It follows from Lemma $5(\mathrm{a})$ that such a vector is a $c$-vector iff $\alpha P_{0}(T-2)=\alpha P_{0}(T)$ and

$$
\alpha P_{i}(T-2)-(n-i+1) P_{i-1}(T)=\alpha P_{i}(T)
$$

holds in $\mathbb{C}[T]$ for $i=1,2, \ldots, n$. The first equation holds iff $P_{0}(T)$ is a constant polynomial, in which case equation (14) holds iff $v$ is a scalar multiple of the vector $\hat{v}$ given in the hypothesis. It is easily verified that $\hat{v}$ satisfies equations (16) and thus is a $c$-vector.

The following corollary states when $M_{\Omega, c}$ has proper submodules.

Corollary 1. $M_{\Omega, c}$ has a proper submodule iff $(2 n-\beta-3)^{2}=1+\gamma$ holds for some positive integer $n$. In this case $U(\mathfrak{g}) \hat{v}$ is a proper submodule, where $\hat{v}$ is the vector given in the hypothesis of Proposition 2.

Proof. This follows immediately from Lemma 4, Proposition 1, and Proposition 2.

\section{REFERENCES}

1. B. Kostant, On Whittaker vectors and representation theory, Invent. Math. 48 (1978), 101184.

2. E. McDowell, On modules induced from Whittaker modules, J. Algebra 96 (1985), 161-177.

3. D. N. Verma, Structure of certain induced representations of complex semi-simple Lie algebras, Bull. Amer. Math. Soc. 74 (1968), 160-166.

Department of Mathematics and Computer Science, Rhode Island College, Providence, RHode IsLAND 02908 